\title{
Thermochemical Nonequilibrium in Rapidly Expanding Flows of High-Temperature Air
}

\author{
Michio Nishida $^{\mathrm{a}}$ and Masashi Matsumoto ${ }^{\mathrm{b}}$ \\ ${ }^{a}$ Department of Aeronautics and Astronautics, Kyushu University, Fukuoka 812-81, Japan \\ ${ }^{b}$ Fluid Dynamics \& Combustion Department, Research Institute, \\ Ishikawajima-Harima Heavy Ind. Co. Ltd., Tokyo 135, Japan
}

Z. Naturforsch. 52a, 358-368 (1997); received June 26, 1996

This paper describes a computational study of the thermal and chemical nonequilibrium occuring
in a rapidly expanding flow of high-temperature air transported as a free jet from an orifice into low-
density stationary air. Translational, rotational, vibrational and electron temperatures are treated
separately, and in particular the vibrational temperatures are individually treated; a multi-vibrational
temperature model is adopted. The governing equations are axisymmetric Navier-Stokes equations
coupled with species vibrational energy, electron energy and species mass conservation equations.
These equations are numerically solved, using the second order upwind TVD scheme of the
Harten-Yee type. The calculations were carried out for two different orifice temperatures and also
two different orifice diameters to investigate the effects of such parameters on the structure of a
nonequilibrium free jet.

Key words: Thermal Nonequilibrium, Chemical Nonequilibrium, High Temperature Air, Free Jet, Computational Fluid Dynamics

\section{Introduction}

High-temperature gas, which is vibrationally excited, partially dissociated and ionized, exhibits thermochemical nonequilibrium when rapidly expanding. In the thermochemical nonequilibrium, thermal and chemical nonequilibrium occurs simultaneously. In the present paper we focus our attention mainly to the thermal nonequilibrium, where there exists nonequilibrium among the translational, rotational, vibrational and electron-translational temperatures. In rapidly expanding flows, the characteristic flow time is in general smaller than the relaxation time for energy exchange between internal energy modes, so that within the expaning flow complete energy exchange will not be established, resulting in thermal nonequilibrium. The rotational temperature is quickly equilibrated with the translational temperature, so that these temperatures may be treated as equal. The vibrational temperature, however, deviates from the translational temperature due to slow equilibration. Moreover, when one treats multi-molecular species,

Reprint requests to Prof. M. Nishida,

e-mail: Nishida@aero.kyushu-u.ac.jp. such species have individual vibrational temperatures, which leads to a multi vibrational temperature model. The electron-translational temperature also deviates from the heavy particle translational temperature and species vibrational temperature.

Such a nonequilibrium rapid-expansion of hightemperature gas is of interest in the following cases:

(i) The generation of thrust in electric propulsion. Electric propulsion, using arc-heated gas, is known as arcjet thruster. For obtaining high thrust, not only translational energy but also vibrational and dissociation energy should be converted as much as possible to directed kinetic energy through an expansion in a thruster nozzle. In the present study we investigate how much vibrational and dissociation energy is converted to thrust.

(ii) Thermal protection of space vehicles. This concerns the heat flux to the surface of a test body. The heat flux depends on the particles in the gas, and the heat produced by their transformations contributes to it.

(iii) Rapidly expanding flows of high-temperature gas are employed to fundamentally study nonequilibrium phenomena. Park and Lee [1] numerically calculated a nozzle flow of high-temperature air, using a 


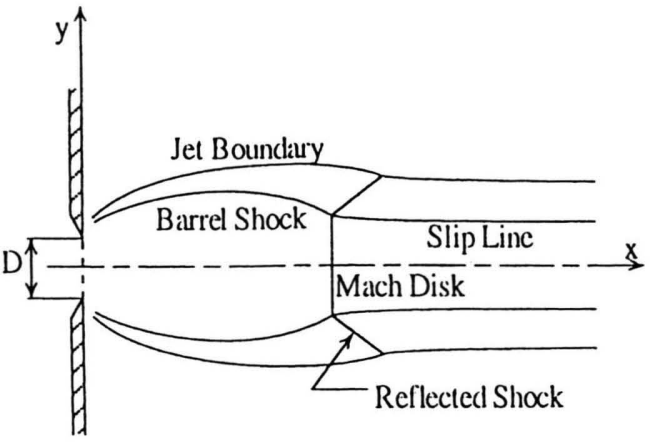

Fig. 1. Structure of a free jet.

multi temperature model in which multi vibrationaltemperatures were considered. According to their calculation, the vibrational temperatures of $\mathrm{N}_{2}, \mathrm{O}_{2}$ and $\mathrm{NO}$, the translational temperature and the electron temperature greatly differ. The vibrational temperatures of $\mathrm{NO}$ and $\mathrm{O}_{2}$ were closer to the translational temperature than the vibrational temperature of $\mathrm{N}_{2}$, and the electron temperature was almost frozen.

In the present paper the free jet expansion is chosen because it is the simplest form of jet flow from which the fundamental nature of the jet flow could be understood. The high-temperature free jet considered here is such that arc-heated air, which is initially in thermal and chemical equilibrium, is expanded from an orifice as a free jet (Figure 1).

\section{Flow Model}

The following assumptions are introduced:

1) The free jet gas is partially dissociated and ionized high-temperature air consisting of the species $\mathrm{N}_{2}, \mathrm{O}_{2}, \mathrm{~N}, \mathrm{O}, \mathrm{NO}, \mathrm{NO}^{+}$and $\mathrm{e}^{-}$. This air is originally composed of $76.5 \% \mathrm{~N}_{2}$ and $23.5 \% \mathrm{O}_{2}$ by mass.

2) Thermal and chemical equilibrium is established upstream of the orifice.

3) An axisymmetric flow is treated, in which viscosity, thermal conduction and diffusion are considered.

4) The flow is in thermal nonequilibrium, however the rotational temperature is fully equilibrated with the translational temperature.

5) The vibrational energy levels of a molecule are populated by a Boltzmann distribution corresponding to the vibrational temperature.

6) All the translational-rotational temperatures of the species are equal.

7) Molecular species have individual vibrational temperatures, that is the multi vibrational temperatures $T_{\text {vib,N }}, T_{\text {vib, }}, T_{\text {vib,NO }}$ and $T_{\text {vib,NO }}$ are considered.

8) The contribution of electronic excitation on the thermodynamic properties can be neglected.

\section{Governing Equations}

The flow field of the free jet in a steady state is numerically calculated by integrating over time the unsteady equations and obtaining the large-time limit. The problem is described by Navier-Stokes equations consisting of mass-, momentum-, overall energy-, species vibrational energy-, electron energy- and species mass conservation equations in unsteady form. Using the cylindrical coordinates given in Fig. 1, the governing equations are written as follows:

$$
\begin{gathered}
\frac{\partial Q}{\partial t}+\frac{\partial F}{\partial x}+\frac{\partial G}{\partial y}+\frac{Z}{y}=\frac{\partial F_{\mathrm{v}}}{\partial x}+\frac{\partial G_{\mathrm{v}}}{\partial y}+\frac{Z_{\mathrm{v}}}{y}+W \\
Q=\left[\begin{array}{c}
\rho \\
\rho u \\
\rho v \\
\rho e \\
\rho_{i} \\
\vdots \\
\rho_{i} e_{\mathrm{vib}, i} \\
\vdots \\
\rho_{\mathrm{e}} e_{\mathrm{e}}
\end{array}\right], F=\left[\begin{array}{c}
\rho u \\
\rho u^{2}+p \\
\rho u v \\
(\rho e+p) u \\
\rho_{i} u \\
\vdots \\
\rho_{i} e_{\mathrm{vib}, i} u \\
\vdots \\
\rho_{\mathrm{e}} e_{\mathrm{e}} u
\end{array}\right], G=\left[\begin{array}{c}
\rho v \\
\rho u v \\
\rho v^{2}+p \\
(\rho e+p) v \\
\rho_{i} v \\
\vdots \\
\rho_{i} e_{\mathrm{vib}, i} v \\
\vdots \\
\rho_{\mathrm{e}} e_{\mathrm{e}} v
\end{array}\right], Z=\left[\begin{array}{c}
\rho v \\
\rho u v \\
\rho v^{2} \\
(\rho e+p) v \\
\rho_{i} v \\
\vdots \\
\rho_{i} e_{\mathrm{vib}, i} v \\
\vdots \\
\rho_{\mathrm{e}} e_{\mathrm{e}} v
\end{array}\right], F_{\mathrm{v}}=\left[\begin{array}{c}
0 \\
\tau_{x x} \\
\tau_{y x} \\
u \tau_{x x}+v \tau_{x y}-q_{x} \\
J_{x, i} \\
\vdots \\
-q_{\mathrm{vib}, x, i} \\
\vdots \\
-q_{\mathrm{e}, x}
\end{array}\right], G_{\mathrm{v}}=\left[\begin{array}{c}
0 \\
\tau_{x y} \\
\tau_{y y} \\
u \tau_{x y}+v \tau_{y y}-q_{y} \\
J_{y, i} \\
\vdots \\
-q_{\mathrm{vib}, y, i} \\
\vdots \\
-q_{\mathrm{e}, y}
\end{array}\right],
\end{gathered}
$$




$$
Z_{\mathrm{v}}=\left[\begin{array}{c}
0 \\
\tau_{x y} \\
2 \mu\left(\frac{\partial v}{\partial y}-\frac{v}{y}\right) \\
u \tau_{x y}+v \tau_{y y}-q_{y} \\
J_{y, i} \\
\vdots \\
-q_{\mathrm{vib}, y, i} \\
\vdots \\
-q_{\mathrm{e}, y}
\end{array}\right], W=\left[\begin{array}{c}
0 \\
0 \\
0 \\
0 \\
\dot{w}_{i} \\
\vdots \\
Q_{\mathrm{T}-\mathrm{v}, i}+Q_{\mathrm{V}-\mathrm{v}, i} \\
+Q_{\mathrm{v}-\mathrm{e}, i}+\dot{e}_{\mathrm{vib}, i} \\
\vdots \\
Q_{\mathrm{e}-\mathrm{T}}+Q_{\mathrm{e}-\mathrm{R}}+Q_{\mathrm{e}-\mathrm{V}}+\dot{e}_{\mathrm{e}}
\end{array}\right]
$$

where $\rho_{i}$ is the density of species $i, \rho$ the density given by $\sum_{i} \rho_{i}, u$ and $v$ are the axial and radial velocities, respectively, $p$ is the pressure, $\tau$ the viscous stress, $\dot{w}_{i}$ the mass production rate of species $i, e$ the total energy, $e_{\text {vib }}$ the vibrational energy, $e_{\mathrm{e}}$ the electron totalenergy, $\dot{e}_{\mathrm{vib}, i}$ the vibrational energy production rate of species $i$ due to chemical reactions, $\dot{e}_{\mathrm{e}}$ the production rate of electron translational-energy due to ionization and recombination, $q_{x_{j}}, q_{\mathrm{vib}, x_{j}, i}$ and $q_{\mathrm{e}, x_{j}}$ are the heat flux, vibrational heat flux and electron heat flux in the $x_{j}$-direction, respectively, $J$ is the diffusion flux, $Q_{\mathrm{T}-\mathrm{V}, i}, Q_{\mathrm{V}-\mathrm{V}, i}, Q_{\mathrm{V}-\mathrm{e}, i}, Q_{\mathrm{e}-\mathrm{T}}$ and $Q_{\mathrm{e}-\mathrm{R}}$ are the energy transfer rates for translation-vibration, vibrationvibration, vibration-electron, electron-translation and electron-rotation, respectively, $\mu$ is the viscosity, and the subscripts $i$, vib and e denote species $i$, vibration and electron, respectively. The total energy $e$ is given by

$$
\begin{aligned}
e=\sum_{i=1}^{N} C_{i} e_{\mathrm{tr}, i} & +\sum_{i=M} C_{i} e_{\mathrm{rot}, i}+\sum_{i=M} C_{i} e_{\mathrm{vib}, i} \\
& +\sum_{i=1}^{N} \frac{C_{i}}{M_{i}} h_{i}^{0}+\frac{1}{2}\left(u^{2}+v^{2}\right),
\end{aligned}
$$

where

$$
\begin{gathered}
e_{\mathrm{tr}, i}=(3 / 2) R_{i} T, \\
e_{\mathrm{rot}, i}=R_{i} T, \\
e_{\mathrm{vib}, i}=R_{i} \theta_{\mathrm{vib}, i} /\left[\exp \left(\theta_{\mathrm{vib}, i} / T_{\mathrm{vib}, i}\right)-1\right] .
\end{gathered}
$$

$C_{i}=\rho_{i} / \rho, M_{i}$ is the molecular weight, $h_{i}^{0}$ the reaction energy, $R_{i}$ the gas constant, $\theta_{\mathrm{vib}, i}$ the characteristic temperature of vibration, $T_{\mathrm{vib}, i}$ is the vibrational temperature, $\sum_{i=M}$ means the summation over the molecular species and $N$ is the total number of species. The heat flux in the $x_{j}$-direction is given by

$$
\begin{gathered}
q_{x_{j}}=-\lambda_{\mathrm{tr}} \frac{\partial T_{\mathrm{tr}}}{\partial x_{j}}-\lambda_{\mathrm{rot}} \frac{\partial T_{\mathrm{tr}}}{\partial x_{j}}-\sum_{i=M} \lambda_{\mathrm{vib}, i} \frac{\partial T_{\mathrm{vib}, i}}{\partial x_{j}} \\
-\lambda_{\mathrm{e}} \frac{\partial T_{\mathrm{e}}}{\partial x_{j}}-\sum_{i}^{N} D_{i} h_{i} \frac{\partial X_{i}}{\partial x_{j}}, \\
q_{\mathrm{vib}, x_{j}, i}=-\lambda_{\mathrm{vib}, i} \frac{\partial T_{\mathrm{vib}, i}}{\partial x_{j}}-D_{i} e_{\mathrm{vib}, i} \frac{\partial X_{i}}{\partial x_{j}}, \\
q_{\mathrm{e}, x_{j}}=-\lambda_{\mathrm{e}} \frac{\partial T_{\mathrm{e}}}{\partial x_{j}}-D_{\mathrm{e}} h_{\mathrm{e}} \frac{\partial X_{\mathrm{e}}}{\partial x_{j}}
\end{gathered}
$$

where $\lambda$ is the heat conductivity, $T_{\mathrm{tr}}$ the translational temperature, $T_{\mathrm{e}}$ the electron temperature, $D_{i}$ the diffusion coefficient, $h_{i}$ the enthalpy, $X_{i}$ the mole fraction, and the subscripts tr and rot denote translation and rotation, respectively.

\section{Properties of High Temperature Air}

\subsection{Transport Properties}

The transport properties (viscosity, thermal conductivity, diffusion coefficient) can be estimated using the Yos' formula [2] which is based on the first Chapman-Enskog approximation.

\subsection{Air Chemistry}

The chemical reactions of air considered in the present work are:

$$
\begin{aligned}
& \mathrm{O}_{2}+M_{1} \rightleftharpoons 2 \mathrm{O}+M_{1}, \\
& \mathrm{~N}_{2}+M_{2} \rightleftharpoons 2 \mathrm{~N}+M_{2}, \\
& \mathrm{~N}_{2}+\mathrm{N} \rightleftharpoons \mathrm{N}, \\
& \mathrm{NO}+M_{1} \rightleftharpoons \mathrm{N}+\mathrm{O}+M_{1}, \\
& \mathrm{NO}+\mathrm{O} \rightleftharpoons \mathrm{O}, \\
& \mathrm{N}_{2}+\mathrm{O} \rightleftharpoons \mathrm{NO}+\mathrm{N}, \\
& \mathrm{N}+\mathrm{O} \rightleftharpoons \mathrm{NO}^{+}+\mathrm{e}^{-},
\end{aligned}
$$

where $M_{1}=\mathrm{O}, \mathrm{N}, \mathrm{O}_{2}, \mathrm{~N}_{2}, \mathrm{NO}$ and $M_{2}=\mathrm{O}, \mathrm{O}_{2}$, $\mathrm{N}_{2}$, NO. The reaction rate constants for the above air chemistry are taken from Park [3].

\subsection{Energy Transfer}

The energy transfers considered here are translation-vibration, translation-electron, rotationelectron, vibration-vibration and vibration-electron as shown in Figure 2. 


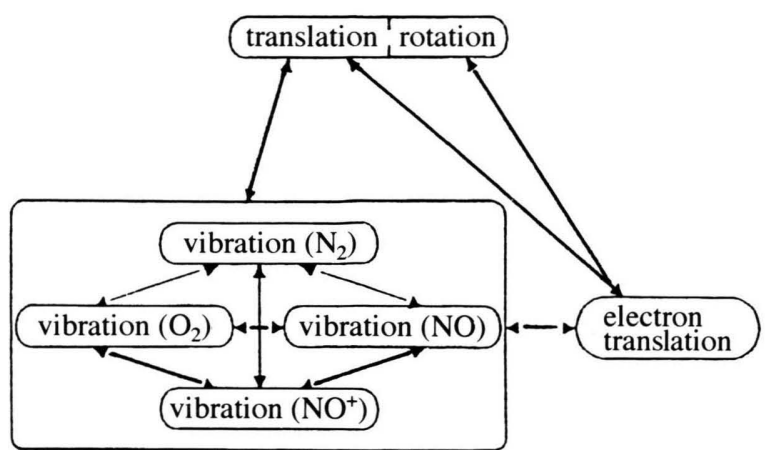

Fig. 2. Internal energy exchange.

\section{Translation-vibration energy transfer}

The energy transfer rate per unit volume between translational and vibrational energy modes is written in the following form:

$$
Q_{\mathrm{T}-\mathrm{V}, i}=\rho \sum_{i=M} C_{i} \frac{e_{\mathrm{vib}, i}^{\mathrm{eq}}-e_{\mathrm{vib}, i}}{\left\langle\tau_{i}\right\rangle},
$$

where

$$
\begin{aligned}
\left\langle\tau_{i}\right\rangle & =\left\langle\tau_{i}^{\mathrm{MW}}\right\rangle / \phi_{i}, \\
e_{\mathrm{vib}, i}^{\mathrm{eq}} & =R_{i} \theta_{\mathrm{vib}, i} /\left[\exp \left(\theta_{\mathrm{vib}, i} / T\right)-1\right] .
\end{aligned}
$$

Vibrational relaxation in an expanding flow is faster than that behind a shock wave, so that MillikanWhite's expression for vibrational relaxation time [4] must be divided by a factor $\phi_{i}$. The value of $\phi_{i}$ was proposed as follows [1]:

$$
\phi_{\mathrm{N}_{2}}=1.5, \phi_{\mathrm{O}_{2}}=1.5, \phi_{\mathrm{NO}}=3, \phi_{\mathrm{NO}^{+}}=3 .
$$

$\left\langle\tau_{i}^{\mathrm{MW}}\right\rangle$ is given by

$$
\left\langle\tau_{i}^{\mathrm{MW}}\right\rangle=\sum_{j} \frac{\rho_{j}}{M_{j}} / \sum_{j} \frac{\rho_{j}}{M_{j} \tau_{i, j}^{\mathrm{MW}}},
$$

where $\tau_{i, j}^{\mathrm{MW}}$ is the vibrational relaxation time of Millikan and White for an $i-j$ encounter [4], given by

$$
\begin{aligned}
\tau_{i, j}^{\mathrm{MW}}= & (p / 101,325)^{-1} \\
& \cdot \exp \left[A_{i, j}\left(T^{-1 / 3}-B_{i, j}\right)-18.42\right] \mathrm{atm} \cdot \mathrm{s},
\end{aligned}
$$

where

$$
\begin{aligned}
A_{i, j} & =1.16 \times 10^{-3} \mu_{i, j}^{1 / 2} \theta_{\mathrm{vib}, i}, \\
B_{i, j} & =0.015 \mu_{i, j}^{1 / 4},
\end{aligned}
$$

where $\theta_{\mathrm{vib}, j}$ is the characteristic temperature of vibration of species $i . \mu_{i, j}$ is the reduced mass of species $i$ and $j$ given by

$$
\mu_{i, j}=10^{3} \frac{M_{i} M_{j}}{M_{i}+M_{j}},
$$

where $M_{i}$ is the molecular weight of species $i$. The coefficients $A_{i, j}$ and $B_{i, j}$ for $\mathrm{N}_{2}-\mathrm{O}, \mathrm{O}_{2}-\mathrm{N}, \mathrm{O}_{2}-\mathrm{O}$ and NO- $j$ encounters are taken from [5]. They are:

$$
\begin{array}{lll}
\mathrm{N}_{2}-\mathrm{O}: & A_{i, j}=72.4, & B_{i, j}=0.0150, \\
\mathrm{O}_{2}-\mathrm{N}: & A_{i, j}=72.4, & B_{i, j}=0.0150, \\
\mathrm{O}_{2}-\mathrm{O}: & A_{i, j}=47.7, & B_{i, j}=0.0590, \\
\mathrm{NO}-j: & A_{i, j}=49.5, & B_{i, j}=0.0420 .
\end{array}
$$

Translation-electron energy transfer

According to Lee [6], the rate of the translational energy transfer between heavy particles and electrons is given by

$$
Q_{\mathrm{T}-\mathrm{e}}=3 \rho_{\mathrm{e}}\left(T-T_{\mathrm{e}}\right)\left(\frac{8 k T_{\mathrm{e}}}{\pi m_{\mathrm{e}}}\right)^{1 / 2} \sum_{i \neq \mathrm{e}} \frac{n_{i} k \sigma_{\mathrm{e}, i}}{m_{i}}
$$

where $\sigma_{\mathrm{e}, i}$ is the cross section for electron- $i$ species encounter. According to Gnoffo et al. [7], $\sigma_{\mathrm{e}, i}$ for the encounters of electron-neutral particle is expressed by the approximation

$$
\sigma_{\mathrm{e}, i}=a_{i}+b_{i} T_{\mathrm{e}}+c_{i} T_{\mathrm{e}}^{2} .
$$

The coefficients $a_{i}, b_{i}$ and $c_{i}$ are:

$$
\begin{array}{lll}
\mathrm{N}_{2}: & a_{i}=7.5 \times 10^{-20}, & b_{i}=5.5 \times 10^{-24}, \\
& c_{i}=-1.0 \times 10^{-28}, & \\
\mathrm{O}_{2}: & a_{i}=2.0 \times 10^{-20}, & b_{i}=6.0 \times 10^{-24}, \\
& c_{i}=0.0, & \\
\mathrm{NO}: & a_{i}=1.0 \times 10^{-19}, & b_{i}=0.0, \\
& c_{i}=0.0, \\
\mathrm{~N}: & a_{i}=5.0 \times 10^{-20}, & \\
& c_{i}=0.0, & \\
\mathrm{O}: & a_{i}=1.2 \times 10^{-20}, & \\
& c_{i}=-2.0 \times 10^{-29} . &
\end{array}
$$


Coulomb cross section is employed for electron-ion encounters.

\section{Rotation-electron energy transfer}

The energy transfer between the rotational and electron energy modes should also be considered in the energy conservation equations because electrons interact with the molecular multipoles produced by deformation of the electron cloud in the formation of molecular bonds [8]. Using the factor $g_{\mathrm{rot}, i}$ (the energy transfer factor between the rotational energy mode and the electron energy mode), the energy transfer rate is written as

$$
Q_{\mathrm{R}-\mathrm{e}}=\sum_{i=M} n_{\mathrm{e}} \frac{2 m_{\mathrm{e}}}{m_{i}} g_{\mathrm{rot}, i} \nu_{\mathrm{e}, i} \frac{3}{2} k\left(T-T_{\mathrm{e}}\right)
$$

The factor $g_{\mathrm{rot}, i}$ is the ratio of $Q_{\mathrm{R}-\mathrm{e}}$ to $Q_{\mathrm{T}-\mathrm{e}}$ for the molecular species $i$. In the present work, we assume $g_{\mathrm{rot}, \mathrm{N}_{2}}=10$ [9]. For oxygen, we set $g_{\mathrm{rot}, \mathrm{O}_{2}}=g_{\mathrm{rot}, \mathrm{N}_{2}}$ because some experimental and theoretical results for oxygen show that the rotational excitation cross section of $\mathrm{O}_{2}$ is similar to that of $\mathrm{N}_{2}$ [10]. The value of $g_{\text {rot,NO }}$ is set to 100 based on the work of Takayanagi and Itikawa [8]. The rotational excitation for moleclar positive ions is governed by the long-range coulomb interaction, so that a large excitation cross section is expected. However, there are no reliable data for this type of excitation, so that in this analysis it is assumed that $g_{\mathrm{rot}, i}$ for a molecular positive ion is equal to that for the corresponding neutral molecule.

\section{Vibration-vibration energy transfer}

Vibration-vibration energy transfer between species $i$ and $j$ is given by [11]

$$
Q_{\mathrm{v}-\mathrm{V}, i}=\sum_{j \neq i} P_{i, j} Z_{i, j} m_{i}\left[e_{\mathrm{vib}, i}\left(T_{\mathrm{vib}, i}^{\prime}\right)-e_{\mathrm{vib}, i}\left(T_{\mathrm{vib}, i}\right)\right],
$$

where $P_{i, j}$ is the collision probability, $T_{\mathrm{vib}, i}^{\prime}$ the same vibrational temperature of two collision partners after the collision for $\mathrm{V}-\mathrm{V}$ energy transfer and $Z_{i, j}$ is the collision number. According to Park and Lee [1],

$$
\begin{aligned}
P_{\mathrm{N}_{2}, \mathrm{NO}} & =5.5 \times 10^{-5}(T / 1000)^{2.32}, \\
P_{\mathrm{N}_{2}, \mathrm{O}_{2}} & =3 \times 10^{-6}(T / 1000)^{2.87}, \\
P_{\mathrm{O}_{2}, \mathrm{NO}} & =P_{\mathrm{N}_{2}, \mathrm{NO}} .
\end{aligned}
$$

In addition, we set

$$
P_{\mathrm{NO}^{+}, i}=P_{\mathrm{N}_{2}, \mathrm{NO}}
$$

\section{Vibration-electronenergy transfer}

The vibration-electron energy transfer rate is given in the conventional form:

$$
Q_{\mathrm{V}-\mathrm{e}, i}=\rho_{i} \frac{e_{\mathrm{vib}, i}^{\mathrm{eq}}\left(T_{\mathrm{e}}\right)-e_{\mathrm{vib}, i}\left(T_{\mathrm{vib}, i}\right)}{\tau_{i-\mathrm{e}}}
$$

The relaxation time $\tau_{\mathrm{N}_{2}-\mathrm{e}}$ is expressed as a function of electron temperature and electron pressure. It was given by (34) and (35) in the work of Lee [12] as follows:

$$
\begin{gathered}
\tau_{\mathrm{N}_{2-\mathrm{e}}}=\frac{1.013 \times 10^{5}}{p_{\mathrm{e}}} 10^{3.91 Z^{2}-30.36 Z+48.90} \\
\text { for } 10^{3} \mathrm{~K} \leq T_{\mathrm{e}} \leq 7 \times 10^{3} \mathrm{~K}, \\
\tau_{\mathrm{N}_{2-\mathrm{e}}}=\frac{1.013 \times 10^{5}}{p_{\mathrm{e}}} 10^{1.30 Z^{2}-9.09 Z+5.85} \\
\text { for } 7 \times 10^{3} \mathrm{~K} \leq T_{\mathrm{e}} \leq 5 \times 10^{4} \mathrm{~K}
\end{gathered}
$$

where

$$
Z=\log _{10}\left(T_{\mathrm{e}} / \mathrm{K}\right) .
$$

The nitrogen-electron coupling is much stronger than the coupling between the electron and other molecules, so that they are expressed as [1]

$$
\begin{gathered}
\tau_{\mathrm{O}_{2}-\mathrm{e}}=300 \tau_{\mathrm{N}_{2}-\mathrm{e}}\left(1.492 T_{\mathrm{e}}\right), \\
\tau_{\mathrm{NO}-\mathrm{e}}=300 \tau_{\mathrm{N}_{2}-\mathrm{e}}\left(1.492 T_{\mathrm{e}}\right) .
\end{gathered}
$$

\section{Method of Numerical Calculation}

A set of governing equations are numerically solved using the second order upwind TVD scheme of the Harten-Yee type [13,14] for the convection terms, and the second order central difference for viscous, heat-conductive, and diffusive terms.

Calculations were done for the upper half region of the free jet $(x / D=8$ and $y / D=4)$, composed of left boundary (orifice and wall), upper boundary (ambient boundary), right boundary (downstream boundary) and lower boundary (free jet axis) (Figure 1). At the orifice, sonic conditions are imposed and a parallel flow is assumed, so that $v=0$. The orifice 
Table 1. Orifice and Ambient Conditions; read $2.599^{-2}$ as $2.599 \times 10^{-2}$.

\begin{tabular}{lcccccccc}
\hline & $T(\mathrm{~K})$ & $p(\mathrm{~atm})$ & $C_{\mathrm{N}_{2}}$ & $C_{\mathrm{O}_{2}}$ & $C_{\mathrm{NO}}$ & $C_{\mathrm{N}}$ & $C_{\mathrm{O}}$ & $C_{\mathrm{NO}^{+}}$ \\
\hline Orifice & 4,000 & 0.53 & $7.456^{-1}$ & $2.599^{-2}$ & $4.050^{-2}$ & $1.114^{-3}$ & $1.868^{-1}$ & $2.953^{-8}$ \\
Ambient & 300 & 0.05 & $7.655^{-1}$ & $2.345^{-1}$ & 0 & 0 & 0 & 0 \\
\hline
\end{tabular}

pressure is set to $p^{*}=0.53 \mathrm{~atm}$. The thermochemical state is assumed to be in equilibrium at the orifice, and the orifice conditions are given in Table 1, where $C_{i}$ denotes the mass fraction of species $i$. No slip conditions, $u=v=0$, and no temperature gradients, $\partial T / \partial x=\partial T_{\mathrm{vib}, i} / \partial x=\partial T_{\mathrm{e}} / \partial x=0$, are put on the wall. Moreover, non-catalytic wall condition $\partial C_{i} / \partial x=0$ is employed. Ambient conditions are given by $u=v=0, T_{\mathrm{tr}}=T_{\mathrm{vib}, i}=T_{\mathrm{e}}=T_{\infty}=300 \mathrm{~K}$ and $p_{\infty}=0.05 \mathrm{~atm}$. Ambient species conditions are shown in Table 1. On the axis, axisymmetric conditions are employed. Outflow conditions are used at the right boundary.

In order to clearly capture the discontinuous plane such as shock wave and slip line, a $160 \times 70$ adaptive grid system was employed, and 10 grid points are put at the orifice. Calculations were carried out for two different orifice temperatures $\left(T^{*}\right), 4,000 \mathrm{~K}$ and $3,000 \mathrm{~K}$, and two different orifice diameters $(D), 1 \mathrm{~cm}$ and $3 \mathrm{~cm}$.

\section{Numerical Results and Discussion}

\subsection{Structure of Nonequilibrium Free Jet}

Figure 3 shows computed temperature contours of internal energy modes for an orifice temperature $T^{*}$ $=4000 \mathrm{~K}$ and an orifice diameter $D=1 \mathrm{~cm}$. Mach disc, barrel shock, jet boundary, reflected shock and slip line can be recognized from a comparison of the translational-temperature contours with the free jet structure shown in Figure 1. The structure of the nonequilibrium free jet is essentially the same as that for a perfect gas, that is to say, the position of the Mach disc for this case is $x / D \simeq 3$, which agrees with the prediction given by Ashkenas and Sherman [15]. However, the result of $T_{\mathrm{vib}, \mathrm{NO}^{+}}$shows a radial spread which is different from the behavior of other temperatures.

Figure 4 shows on-axis temperature profiles. The translational temperature is decreased up to $x / D \simeq 3$ and then suddenly elevated due to the compression by the Mach disc. The region from the orifice to
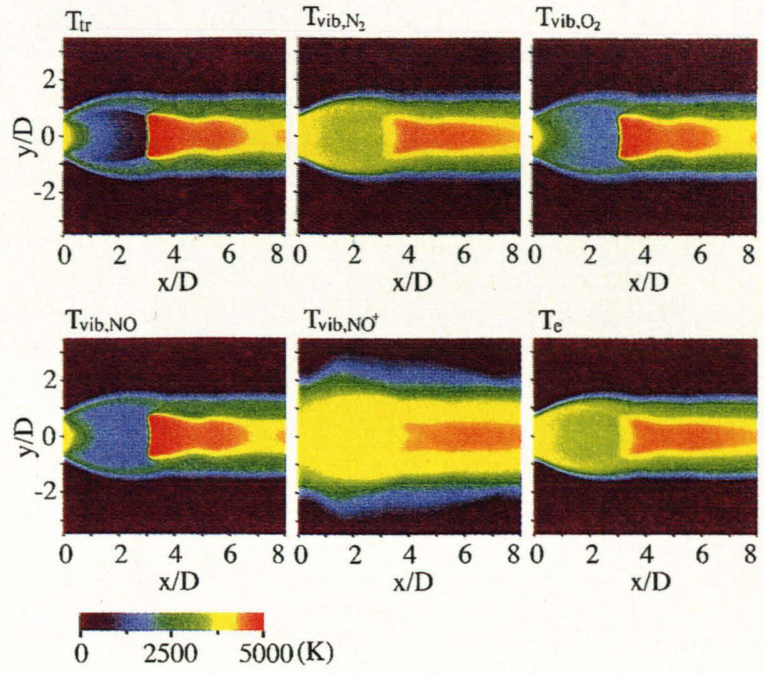

Fig. 3. Temperature contours for $T^{*}=4000 \mathrm{~K}, p^{*}=0.528$ atm and $D=1 \mathrm{~cm}$.

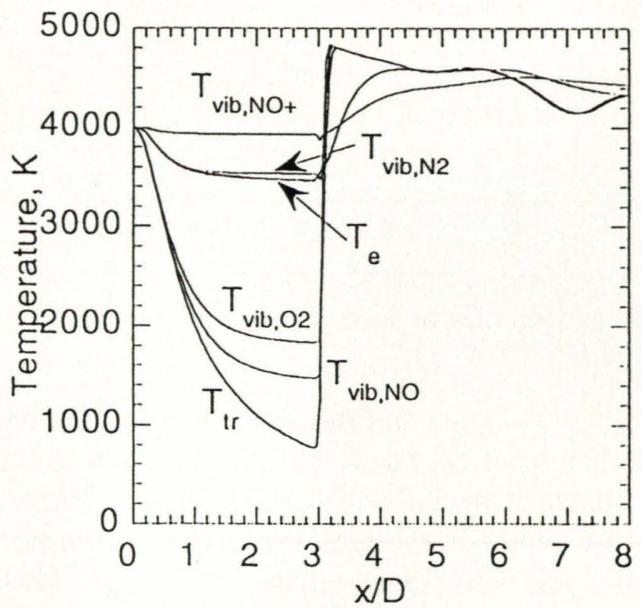

Fig. 4. On-axis profiles of temperature for $T^{*}=4000 \mathrm{~K}$ and $D=1 \mathrm{~cm}$.

this Mach disc is recognized as supersonic expansion region. $T_{\mathrm{vib}, \mathrm{O}_{2}}$ and $T_{\text {vib,NO }}$ remain in equilibrium with $T_{\text {tr }}$ up to $x / D \simeq 0.5$ and then deviate from it. Nevertheless, these two vibrational temperatures are closer to $T_{\text {tr }}$ than other vibrational tempera- 


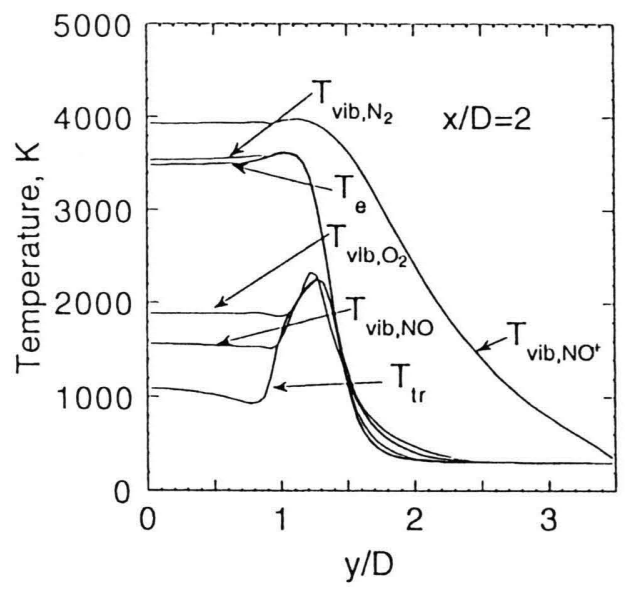

Fig. 5. Radial profiles of temperature for $T^{*}=4000 \mathrm{~K}$ and $D=1 \mathrm{~cm}$.

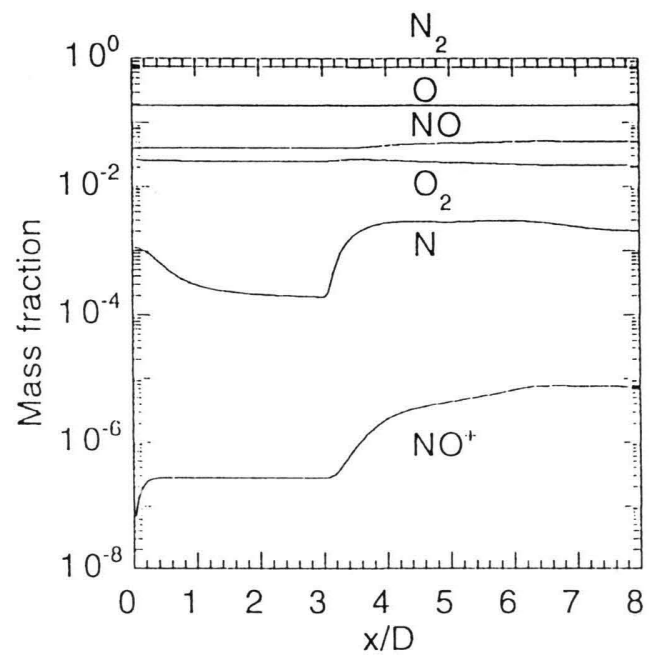

Fig. 6. On-axis profiles of species mass fraction for $T^{*}=$ $4000 \mathrm{~K}$ and $D=1 \mathrm{~cm}$.

tures $\left(T_{\mathrm{vib}, \mathrm{N}_{2}}, T_{\mathrm{vib}, \mathrm{NO}^{+}}\right)$and the electron temperature. On the other hand, $T_{\mathrm{vib}, \mathrm{NO}^{+}}, T_{\mathrm{vib}, \mathrm{N}_{2}}$ and $T_{\mathrm{e}}$ become rapidly frozen; especially $T_{\mathrm{vib}, \mathrm{NO}^{+}}$is almost frozen in the entire supersonic-region. Surprisingly, the behavior of $T_{\mathrm{e}}$ is very close to that of $T_{\mathrm{vib}, \mathrm{N}_{2}}$. This is owing to the very strong coupling of $\mathrm{N}_{2}$ vibration and electron-translation; the energy transfer rate between $\mathrm{N}_{2}$-vibration and electron-translation is 300 times larger than those between other vibrations and electron-translation. The results from the numerical analysis of hight-temperature expansion flows done by Park and Lee [1] shows that the nearest vibrational temperature to translational temperature is $T_{\mathrm{vib}, \mathrm{NO}}$, and the next one is $T_{\text {vib, } \mathrm{O}_{2}}$. They did not estimate $T_{\text {vib,NO+ }}$, so that the highest vibrational temperature was $T_{\mathrm{vib}, \mathrm{N}_{2}}$. The results of vibrational temperature obtained by them are very similar to the present ones. However, their computation also indicated that the electron temperature was considereably higher than the vibrational temperature of $\mathrm{N}_{2}$. This result differs much from the present result that $T_{\mathrm{e}}$ is close to $T_{\text {vib, } \mathrm{N}_{2}}$ due to the rapid energy transfer between the $\mathrm{N}_{2}$-vibrational and the electron-translational energy mode.

Radial profiles of temperature at $x / D=2$ are shown in Figure 5. $T_{\text {tr }}$ is decreased with radial distance and then increased by a barrel shock. It is decreased again towards outside, which shows a jet boundary. $T_{\mathrm{vib}, \mathrm{O}_{2}}$ and $T_{\text {vib,NO }}$ also illustrate a profile similar to $T_{\mathrm{tr}}$. $T_{\mathrm{vib}, \mathrm{N}_{2}}$ and $T_{\mathrm{e}}$ show an appreciable increase due to a compression by the barrel shock, while $T_{\text {vib, } \mathrm{NO}^{+}}$has a very different radial profile from the other temperatures.

On-axis profiles of species mass fraction are shown in Figure 6. It is seen that the mass fractions of $\mathrm{N}_{2}$, $\mathrm{O}, \mathrm{NO}$ and $\mathrm{O}_{2}$ do not change up to the Mach disc $(x / D \simeq 3)$ and in particular the mass fractions of $\mathrm{N}_{2}$ and $\mathrm{O}$ show constant profiles in the entire region. It may be mentioned from these results that the chemical reactions related to the main four species $\left(\mathrm{N}_{2}, \mathrm{O}, \mathrm{NO}\right.$, $\mathrm{O}_{2}$ ) are almost frozen in the supersonic region from the orifice to the Mach disc, which is consistent with the results of Park and Lee [1].

The mass fraction of $\mathrm{N}$ is decreased from the orifice to the Mach disc, which implies that chemical reactions decreasing $\mathrm{N}$, namely recombinations into $\mathrm{N}_{2}$, take place in a rapid expansion. It is increased across the Mach disc due to dissociation into N. The mass fraction of $\mathrm{NO}^{+}$rapidly increases immediately downstream of the orifice and then shows a constant profile up to the Mach disc. The mass fractions of $\mathrm{N}$ and $\mathrm{NO}^{+}$are very small compared with those of $\mathrm{N}_{2}$ and $\mathrm{NO}$, so that $\mathrm{N}_{2}$ and $\mathrm{NO}$ are not influenced by $\mathrm{N}$ and $\mathrm{NO}^{+}$.

\subsection{Comparison of Results for Single Vibrational- Temperature and Multi Vibrational-Temperature Models}

A numerical calculation for a two-temperature model $\left(T_{\mathrm{tr}}\right.$ and $\left.T_{\mathrm{vib}}\right)$ using single vibrational-temperature $\left(T_{\text {vib, } \mathrm{N}_{2}}=T_{\text {vib }, \mathrm{O}_{2}}=T_{\mathrm{vib}, \mathrm{NO}}=T_{\text {vib }, \mathrm{NO}^{+}}=T_{\text {vib }}\right)$ was carried out to be compared with the results for 

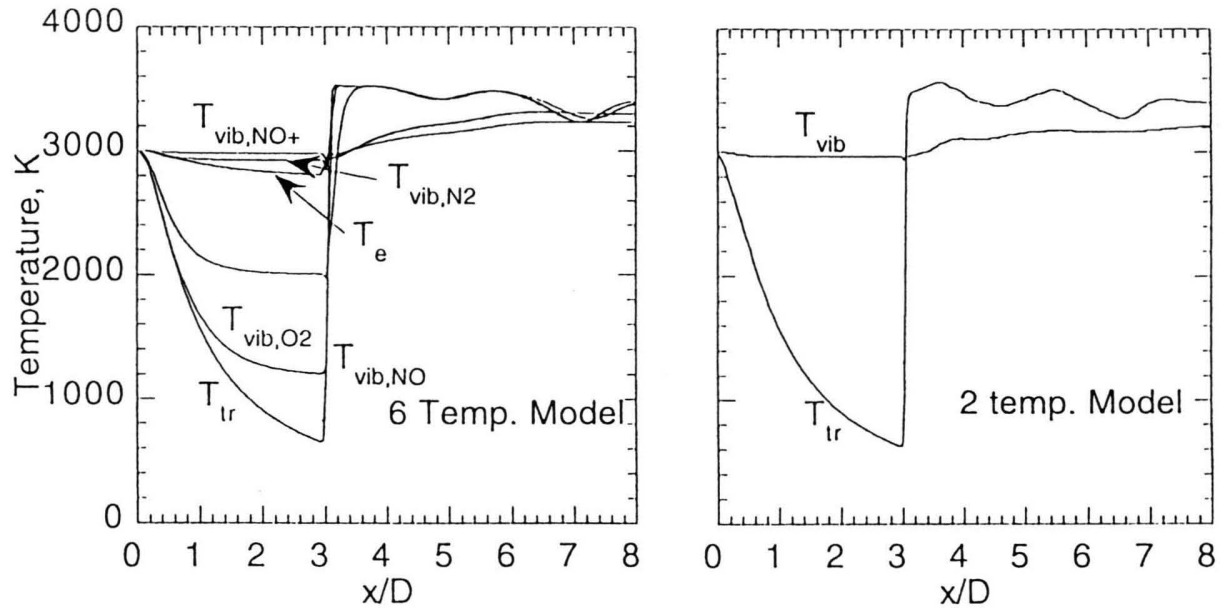

Fig. 7. On-axis temperature profiles for a six temperature model and two temperature model, $T^{*}=3000 \mathrm{~K}$ and $D=1 \mathrm{~cm}$.
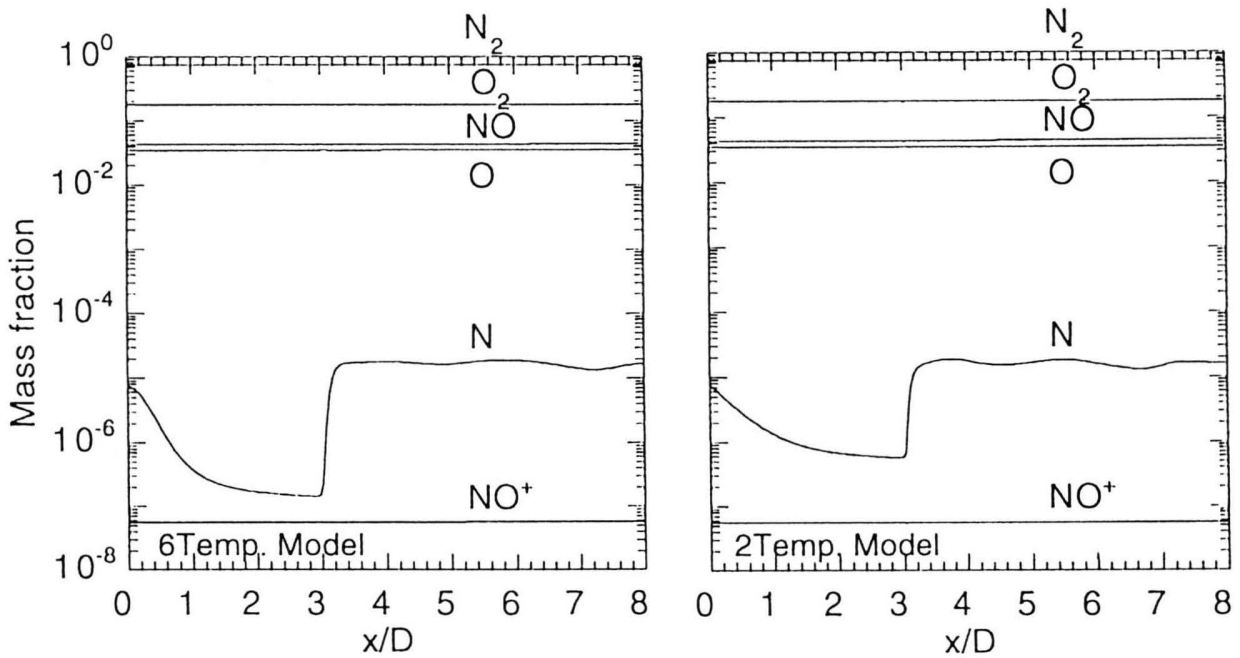

Fig. 8. On-axis profiles of species mass fraction for a six temperature model and two temperature model, $T^{*}=3000 \mathrm{~K}$ and $D=1 \mathrm{~cm}$.

the six-temperature model using multi vibrationaltemperatures. In this calculation, the electron temperature was neglected because the degree of ionization is less than $10^{-5}$, as seen in Fig. $6^{*}$, which leads to a negligible effect of electrons on the flow field. A comparison of on-axis temperature profiles for the sixtemperature model and the two-temperature model is illustrated in Fig. 7, where the orifice conditions were set to $T^{*}=3000 \mathrm{~K}$ and $D=1 \mathrm{~cm}$. It is seen from the comparison that the vibrational temperature for the two temperature model is very similar to $T_{\mathrm{vib}, \mathrm{N}_{2}}$ for the multi vibrational-temperature model. Moreover,

${ }^{*}$ Single ionization is considered here, so that $n_{\mathrm{NO}^{+}}=n_{\mathrm{e}}$. the translational temperatures in both cases are the same.

On-axis mass fractions in both cases are given in Figure 8 . There are no differences between these cases except that the mass fraction profiles of $\mathrm{N}$ are different in the supersonic region up to $x / D \simeq 3$. It may be mentioned that the two temperature model is sufficient for the investigation of the mass fractions of the main species $\mathrm{N}_{2}, \mathrm{O}_{2}$, $\mathrm{NO}$ and $\mathrm{N}$.

\subsection{Effect of Orifice Size}

In order to clarify the effect of size of the orifice on the thermal nonequilibrium, a calculation for an 


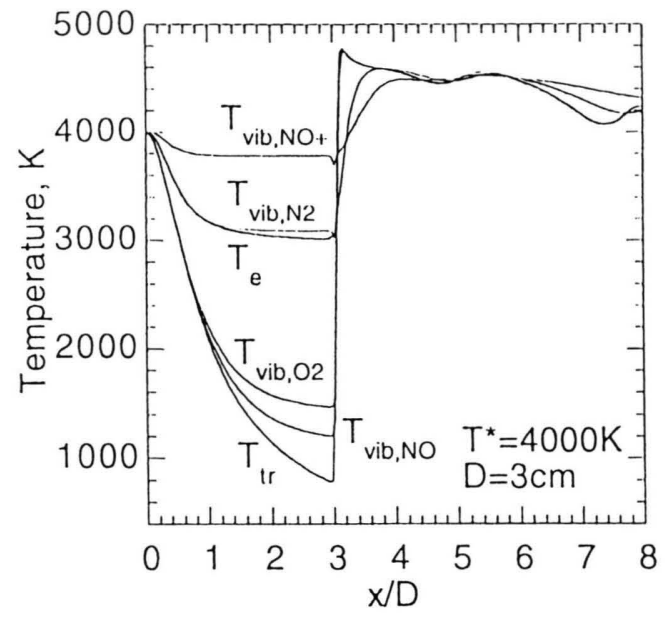

Fig. 9. On-axis profiles of temperature, $T^{*}=4000 \mathrm{~K}$ and $D=3 \mathrm{~cm}$.

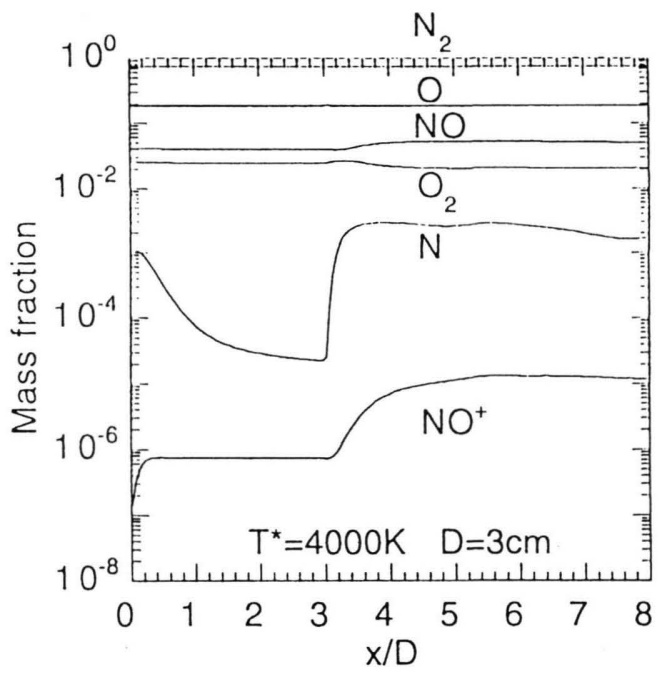

Fig. 10. On-axis profiles of species mass fraction, $T^{*}=4000 \mathrm{~K}$ and $D=3 \mathrm{~cm}$.

orifice diameter $D=3 \mathrm{~cm}$ was performed, and the result, shown in Figs. 9 and 10, can be compared with the result for $D=1 \mathrm{~cm}$, shown in Figs. 4 and 6. It is seen that the decreases of all vibrational temperatures are larger for the larger diameter. This result is reasonable, because the flow goes to equilibrium when the ratio of the characteristic flow time (the ratio of reference length to reference velocity) to the relaxation time for $\mathrm{T}-\mathrm{V}$ energy transfer becomes larger. It is also remarkable that the extent of a decrease in mass fraction of $\mathrm{N}$ in the supersonic region up to $x / D \simeq 3$

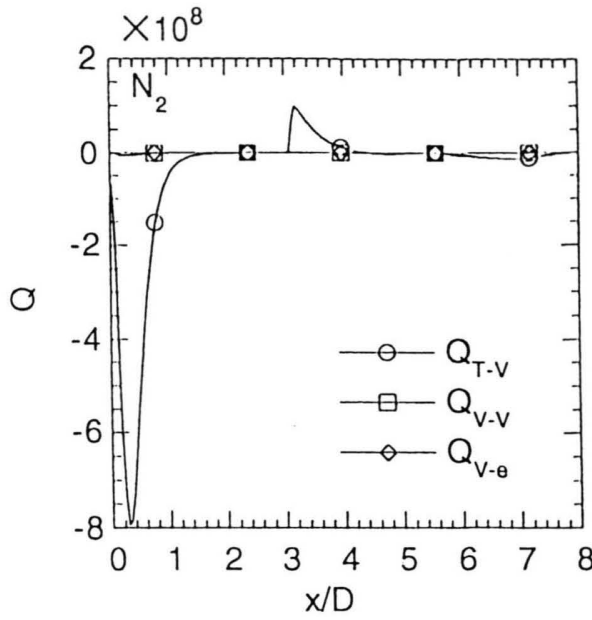

Fig. 11. Energy transfer rates for $\mathrm{N}_{2} \cdot Q$ in the units of $\mathrm{W} / \mathrm{m}^{3}$.

is larger in the case of larger orifice size than in the smaller one.

\subsection{Effect of Orifice Temperature}

The effect of orifice temperature on thermal nonequilibrium can be understood from a comparison of the results shown in Fig. $4\left(T^{*}=4000 \mathrm{~K}\right)$ and $7\left(T^{*}=3000 \mathrm{~K}\right)$. When the orifice temperature is higher, vibrational temperatures come closer to the translational temperature. This result can be explained as follows: When the speed of sound at the orifice is chosen as reference velocity, the characteristic flow time is inversely proportional to $\sqrt{T^{*}}$. The relaxation time for T-V energy transfer is inversely proportional to $T^{*}$. Then the ratio of the characteristic flow time to the relaxation time for energy transfer is increased with orifice temperature which goes to an equilibrium flow.

\subsection{Energy Transfer Rates}

In Fig. 11 are shown the on-axis profiles of the energy transfer rates for $\mathrm{N}_{2}$, i. e., $Q_{\mathrm{T}-\mathrm{V}, \mathrm{N}_{2}}, Q_{\mathrm{V}-\mathrm{V}, \mathrm{N}_{2}}$, $Q_{\mathrm{V}-\mathrm{e}, \mathrm{N}_{2}}$ in units of $\mathrm{W} / \mathrm{m}^{3}$. It appears that the dominant energy transfer is between translation and vibration $(\mathrm{T}-\mathrm{V})$, and that other energy transfers are negligible. The T-V energy transfer is significant in the supersonic region from the orifice to the Mach disc, showing a decreasing and then increasing profile. This means that the vibrational energy of $\mathrm{N}_{2}$ is 


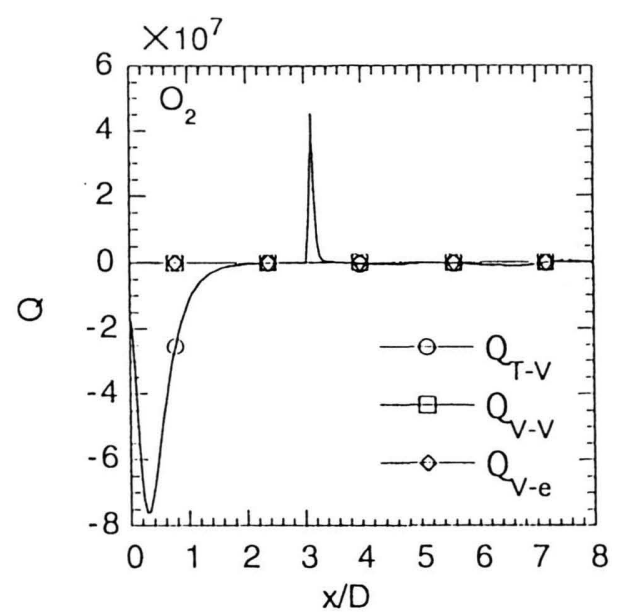

Fig. 12. Energy transfer rates for $\mathrm{O}_{2}, Q$ in the units of $\mathrm{W} / \mathrm{m}^{3}$.

transferred to translational energy, which is consistent with $T_{\text {vib, } \mathrm{N}_{2}}>T_{\text {tr }}$ in this region. In the subsonic region behind the Mach disc, an increase from zero in $Q_{\mathrm{T}-\mathrm{V}, \mathrm{N}_{2}}$ is seen in Fig. 11, which is caused by the energy transfer from the translational energy mode to the vibrational energy mode.

Figures 12 and 13 illustrate the on-axis energy transfer rates for $\mathrm{O}_{2}$ and $\mathrm{NO}$, respectively. For these two species, the T-V energy transfer is also dominant, and it occurs in the same regions as for $\mathrm{N}_{2}$, other energy transfer rates being negligible. However, it is interesting that the V-V energy transfer for NO appreciably appears immediately downstream of the orifice. It may be mentioned that the T-V energy transfer is the most dominant one in the nonequilibrium expansion flow treated here.

\section{Concluding Remarks}

Thermal and chemical nonequilibrium free jets of high-temperature air, which are initially in equilibrium upstream of an orifice and are expanded from the orifice to low-density, were numerically analyzed by using a six temperature model consisting of translational, electron and vibrational temperatures of $\mathrm{N}_{2}$, $\mathrm{O}_{2}$, NO and $\mathrm{NO}^{+}$, whereby the thermal and chemical nonequilibrium in a rapidly expanding flow was clarified. The present results indicate not only that the vibrational temperatures greatly deviate from translational temperature but also that the vibrational temperatures are out of equilibrium with each other. Of four vibrational temperatures, the coupling of $T_{\mathrm{vib}, \mathrm{NO}}$ and $T_{\text {tr }}$ is comparatively strong, whereas the vibrational

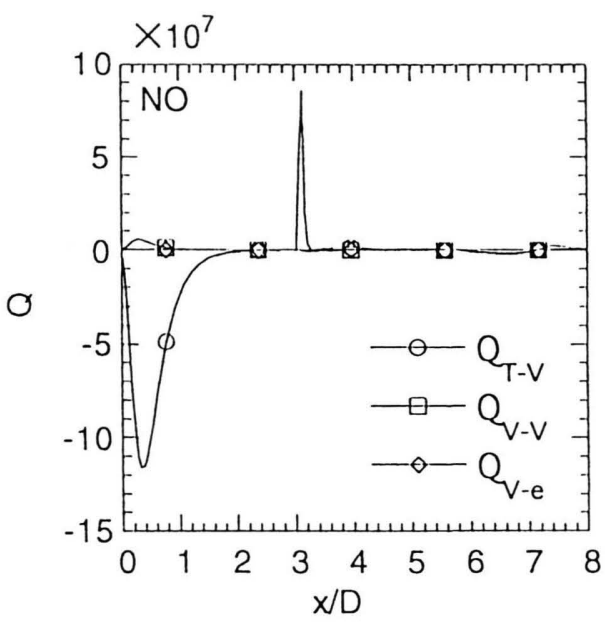

Fig. 13. Energy transfer rates for NO. $Q$ in the units of $\mathrm{W} / \mathrm{m}^{3}$.

temperature of the most dominant species $\mathrm{N}_{2}$ is significantly different from $T_{\text {tr }}$ in the supersonic region. If the behavior of $T_{\mathrm{vib}, \mathrm{N}_{2}}$ is considered in a nozzle of an arcjet thruster using nitrogen gas as propellant, it may be mentioned that the vibrational energy of $\mathrm{N}_{2}$ will not contribute to thrust. It was clarified that the electron temperature is very close to the vibrational temperature of $\mathrm{N}_{2}$ due to a very strong coupling between them.

Chemical nonequilibrium was also investigated. The on-axis profiles of species mass fractions indicate that the main four species, $\mathrm{N}_{2}, \mathrm{O}_{2}, \mathrm{O}$ and $\mathrm{NO}$, show a chemically frozen expansion flow not only in the supersonic region but also in the subsonic region downstream of the Mach disc. On the other hand, the mass fraction of $\mathrm{N}$ is decreased from the orifice to the Mach disc and then increased immediately behind the Mach disc. This implies that $\mathrm{N}$ is recombined in the supersonic region, and dissociation into $\mathrm{N}$ occurs behind the Mach disc. The mass fraction of $\mathrm{NO}^{+}$is also increased downstream of the Mach disc. This is caused by the ionization process $\mathrm{N}+\mathrm{O} \rightarrow \mathrm{NO}^{+}+\mathrm{e}^{-}$.

The results for the six-temperature model were compared with those for the two temperature model. According to this comparison, the vibrational temperature for the latter case is nearly the same as that of $\mathrm{N}_{2}$ for the former case. Moreover, the translational temperature is not influenced by the temperature model.

The effects of orifice size and orifice temperature on the nonequilibrium free jet were investigated. It 
was clarified that the vibrational temperatures come close to the translational temperature if the orifice diameter is large and temperature of the orifice is high.

[1] C. Park and S.-H. Lee, J. Thermophys. and Heat Transfer 9, 9 (1995).

[2] J. M. Yos, Technical Memorandum RAD TM-63-7, AVCO-RAD, 1963.

[3] C. Park, Progress in Astronautics and Aeronautics 103, AIAA, 478 (1985).

[4] R. C. Millikan and D. R. White, J. Chem. Phys. 39, 3209 (1963).

[5] C. Park, J. Thermophys. and Heat Transfer 7, 385 (1993).

[6] J.-H. Lee, Progress in Astronautics and Aeronautics 96, 3 (1984).

[7] P. A. Gnoffo, R. N. Gupta and J. L. Shin, NASA TP2867, 1989.

[8] K. Takayanagi and Y. Itikawa, Advances in Atomic
Finally, the on-axis energy transfer rates have been investigated for $\mathrm{N}_{2}, \mathrm{O}_{2}$ and $\mathrm{NO}$, showing that the $\mathrm{T}-\mathrm{V}$ energy transfer is dominant and the other types of energy transfer play no important role.

and Molecular Physics, Vol.6, Academic Press, New York 1970, p. 105.

[9] S. Lazdinis and S. L. Petrie, Phys Fluids 17, 1539 (1974).

[10] H. S. W. Massey, E. H. S. Burhop and H. B. Gilbody, Electronic and Ionic Impact Phenomena, Vol.2, 2nd ed. Oxford Univ. Press, 1967, p. 350.

[11] G. V. Candler and R. W. MacCormack, J. Thermophys. and Heat Transfer 5, 266 (1991).

[12] J.-H. Lee, J. Thermophys. and Heat Transfer 7, 399 (1993).

[13] A. Harten, J. Comp. Phys. 49, 357 (1983).

[14] H. C. Yee, NASA TM-101088, 1989.

[15] H. Ashkenas and F. S. Sherman, Rarefied Gas Dynamics, Academic Press, New York 1965, p. 84. 